\title{
Applications of Inorganic Nanomaterials in Photothermal Therapy Based on Combinational Cancer Treatment
}

This article was published in the following Dove Press journal: International Journal of Nanomedicine

Ji Wang, ${ }^{1, *}$ Xia $\mathrm{Wu}^{2, *}$ Peng Shen, ${ }^{3}$ Jun Wang, ${ }^{4}$ Yidan Shen, ' Yan Shen, ${ }^{5}$ Thomas J Webster, (1D ${ }^{6}$ Junjie Deng ${ }^{3}$

'Department of Oncology, The Second Affiliated Hospital of Soochow University, Suzhou, People's Republic of China; ${ }^{2}$ Department of Clinical Pharmacology, The Second Affiliated Hospital of Soochow University, Suzhou, People's Republic of China; ${ }^{3}$ Engineering Research Center of Clinical Functional Materials and Diagnosis \& Treatment Devices of Zhejiang Province, Wenzhou Institute, University of Chinese Academy of Sciences, Wenzhou, People's Republic of China; ${ }^{4}$ Department of General Surgery, The Fifth People's Hospital of Wujiang, Suzhou, People's Republic of China; ${ }^{5}$ Department of Pharmaceutics, China Pharmaceutical University, Nanjing, People's Republic of China; ${ }^{6}$ Department of Chemical Engineering, Northeastern University, Boston, MA, USA

*These authors contributed equally to this work

Correspondence: Junjie Deng

Wenzhou Institute, University of Chinese Academy of Sciences, No. 16 Xinsan Road, Hi-Tech Industry Park, Wenzhou, Zhejiang, People's Republic of China

Tel +86 57788017548

Fax +86 57788017554

Email j.deng@wibe.ac.cn

Thomas J Webster

Department of Chemical Engineering,

Northeastern University, 360 Huntington

Avenue, Boston, MA 02115, USA

Tel + I 6173736585

Email th.webster@neu.edu
Background: Cancer is one of the major causes of death and is difficult to cure using existing clinical therapies. Clinical cancer treatments [such as surgery, chemotherapy (CHT), radiotherapy (RT) and immunotherapy (IT)] are widely used but they have limited therapeutic effects and unavoidable side effects. Recently, the development of novel nanomaterials offers a platform for combinational therapy (meaning a combination of two or more therapeutic agents) which is a promising approach for cancer therapy. Recent studies have demonstrated several types of nanomaterials suitable for photothermal therapy (PTT) based on a near-infrared (NIR) light-responsive system. PTT possesses favorable properties such as being low in cost, and having high temporospatial control with minimal invasiveness. However, short NIR light penetration depth limits its functions.

Methods: In this review, due to their promise, we focus on inorganic nanomaterials [such as hollow mesoporous silica nanoparticles (HMSNs), tungsten sulfide quantum dots ( $\left.\mathrm{WS}_{2} \mathrm{QDs}\right)$, and gold nanorods (AuNRs)] combining PTT with CHT, RT or IT in one treatment, aiming to provide a comprehensive understanding of PTT-based combinational cancer therapy.

Results: This review found much evidence for the use of inorganic nanoparticles for PTTbased combinational cancer therapy.

Conclusion: Under synergistic effects, inorganic nanomaterial-based combinational treatments exhibit enhanced therapeutic effects compared to PTT, CHT, RT, IT or PDT alone and should be further investigated in the cancer field.

Keywords: photothermal agents, hyperthermia, chemotherapy, radiotherapy, immunotherapy, photodynamic therapy

\section{Introduction}

As a leading cause of death, cancer is one of the most serious public health problems worldwide. According to current statistics, it is projected that more than 1.7 million new cases and 0.6 million cancer deaths will occur in the United States in 2019 alone. ${ }^{1}$ Cancer is not a particular disease but a group of diseases and alarmingly there are more than one hundred different types of cancers. Moreover, cancer is hard to cure because of the high mutation rate and metastasis of cancer cells. Current main cancer treatments include surgery, CHT, RT and IT. It is common to ablate tumors as the first treatment, but it is difficult to remove tumor tissues completely through surgery. Even a single residual cancer stem cell can grow into a new tumor. The remaining cancer cells can be killed via CHT, however, patients have to suffer serious side effects caused by intrinsic off-target toxicity of CHT drugs. ${ }^{2}$ RT can kill cancer cells and shrink tumors with high 
doses of radiation, but it can also damage nearby healthy cells. IT treats cancer by boosting the immune system against cancer cells, but it often happens that the immune system acts against healthy cells and tissues. Besides the above side effects, there is one more important problem - infection. Cancer patients are susceptible to infections due to their weakened immune systems. The problem can become worse after the immune system suffers more damage from cancer treatments such as surgery, CHT and RT. The use of antibiotics can solve infections, but it can also induce new problems like drug resistance bacteria.

Although the above therapies are not effective all the time and have unavoidable side effects, they still comprise the primary method of clinical cancer treatment. The development of nanotechnology has led to the development of new nanomaterials with several attractive properties providing a promising novel way for combining various cancer treatments together. ${ }^{3}$ Firstly, nanomaterials can be used as carriers to encapsulate drugs to prolong drug plasma circulation time and reduce contact with normal cells. In other words, nanomaterials can increase the efficiency of the drugs to target cancer cells and alleviate off-target toxicities. ${ }^{4}$ Secondly, nanoparticles can easily accumulate in tumors via the enhanced permeability and retention (EPR) effects caused by leaky tumor vasculature and dysfunctional lymphatic drainage. ${ }^{2}$ This feature is the result of unintended coordination between solid tumors and nanoparticles, but it helps to improve drug deposition and target tumors. ${ }^{5}$

Thirdly, there is a wide range of diverse nanomaterials available today. Nanomaterials are roughly separated into inorganic and organic materials. Inorganic nanomaterials include examples of gold nanoparticles (AuNP), ${ }^{6}$ mesoporous silica nanoparticles (MSNP), ${ }^{7}$ quantum dots (QD), carbon nanotubes $(\mathrm{CNT}),{ }^{8}$ etc. Moreover, common organic nanomaterials include liposomes, polymeric micelles, dendrimers, etc. Due to their greater surface area, nanomaterials have unique properties that can play a role in cancer treatment regimes as enhanced photosensitizers for PDT. Fourthly, nanoparticles provide a platform for cancer treatment with unlimited possibilities according to each unique nanomaterial design. Besides the type of nanomaterial, the pharmacokinetics and biodistribution of nanoparticles can also be attributed to properties such as size, shape and surface modification. ${ }^{9,10}$ Nanoparticles with sizes in the range of 5 100 $\mathrm{nm}$ have a higher chance to survive renal clearance so that they have a great circulation time for accumulating in tumors. ${ }^{11}$ As one of the most common nanoparticle surface modifications, PEGylation, improves nanoparticle pharmacokinetic properties by creating a hydrophilic layer resistant to protein adsorption and immune system clearance. ${ }^{12}$ Finally, nanomaterials can possess excellent antibacterial activity to protect cancer patients from infections. ${ }^{13}$ Several nanoparticles have been proven to be effective to treat infectious diseases, even antibiotic resistant ones, in vitro and in vivo. ${ }^{14}$ Such studies highlight that the use of antibacterial nanomaterials can help reduce the abuse of antibiotics, and thus the emergence of antibiotic resistant bacteria.

There are several novel nanomaterial-based stimuliresponsive systems for cancer treatment (Table 1). According to tumor properties (such as type, size and

Table I Novel Nanomaterial-Based Stimuli-Responsive Systems for Cancer Treatment

\begin{tabular}{|c|c|c|c|}
\hline Nanomaterial & Mechanism & Therapy & Ref \\
\hline $\mathrm{SCNs} / \mathrm{Pt}$ & $\mathrm{pH}$-responsive size switching & $\mathrm{CHT}$ & [16] \\
\hline DOX/CP-NI NPs & Light-triggered ROS generation; hypoxia-responsive chemo drug release & $\mathrm{PDT} / \mathrm{CHT}$ & [17] \\
\hline $\mathrm{PhA} / \mathrm{AuNPs}$ & Light-triggered GSH-responsive ROS generation & PDT & [18] \\
\hline $\begin{array}{l}\text { TMPyP\&DNM\&Apt-gc34 } \\
\text { @SPION }\end{array}$ & $\begin{array}{l}\text { Light-triggered } \mathrm{ROS} \text { generation; magnetic targeted } \mathrm{pH} \text {-responsive chemo drug } \\
\text { release }\end{array}$ & $\mathrm{PDT} / \mathrm{CHT}$ & [21] \\
\hline SPION/DOX/Microbubble & Ultrasound/magnetic targeted chemo drug delivery & $\mathrm{CHT}$ & [24] \\
\hline BPQDs/PLGA nanospheres & Light-triggered hyperthermia & PTT & [25] \\
\hline HPSN-Pax/PdPc & Light-triggered hyperthermia and chemo drug release & $\mathrm{PTT} / \mathrm{CHT}$ & [26] \\
\hline mPEG@HGNPs & Light-triggered hyperthermia; X-ray radiation & $\mathrm{PTT} / \mathrm{RT}$ & [27] \\
\hline HCuSNPs-CpG & Light-triggered hyperthermia; stimulating immune system & $\mathrm{PTT} / \mathrm{IT}$ & [28] \\
\hline UCNPs-NGO/ZnPc & Light-triggered hyperthermia and ROS generation & PTT/PDT & [29] \\
\hline
\end{tabular}

Abbreviations: SCNs, pH-sensitive cluster nanobombs; Pt, platinum; DOX, doxorubicin; CP-NI, 2-nitroimidazole-grafted conjugated polymer; ROS, reactive oxygen species; PhA, pheophorbide a; TMPyP, 5, 10,I5,20-tetrakis (I-methylpyridinium-4-yl) porphyrin; DMN, daunomycin; SPION, superparamagnetic iron oxide nanoparticles; BPQDs, black phosphorus quantum dots; PLGA, poly (lactic-co-glycolic acid); HPSN, hollow structured polymer-silica nanohybrid; Pax/PdPc, paclitaxel and palladium phthalocyanine; HGNPs, hollow gold nanoparticles; HCuSNPs, hollow CuS nanoparticles; CpG, cytosine-guanine; UCNPs, upconversion nanoparticles; NGO, nanographene oxide; ZnPc, zinc phthalocyanine. 
location), various internal and external stimuli have been selected for site-specific therapy. ${ }^{15}$ Internal stimuli take advantage of tumor-specific microenvironments including low $\mathrm{pH},{ }^{16}$ hypoxia, ${ }^{17}$ high levels of glutathione $(\mathrm{GSH})^{18}$ and over-expressed cancer-associated enzymes. ${ }^{19}$ For example, Li et al developed $\mathrm{pH}$-responsive nanoparticles which can keep the nanoparticle size to $100 \mathrm{~nm}$ in normal $\mathrm{pH}$ conditions and change it to $5 \mathrm{~nm}$ at low $\mathrm{pH}$ conditions of the tumor microenvironment. The initial size prolongs plasma circulation time of the nanoparticle and the final size improves their tissue penetration and extravasation. ${ }^{15}$ However, it is difficult to precisely control an internal stimuli-responsive system because of complex microenvironments and individual variations in the body. ${ }^{20}$

Compared to internal stimuli, external stimuli possess more advantages on the temporal and spatial control of nanoparticles with minimal invasiveness. Here, the nanoparticle system is not activated until the appearance of an external stimulus such as light, magnetic field irradiation and ultrasound. ${ }^{2}$ Magnetic field technology has recently received much attention for magnetic hyperthermia, magnetic resonance imaging and a controlled drug release system. For example, Sun's team developed a multifunctional nanocarrier of super paramagnetic iron oxide nanoparticles (SPION) for delivering CHT agents and photosensitizer molecules to the tumor site. The delivery system displayed controlled drug release to cancer microenvironments. ${ }^{21}$ Ultrasound is also an excellent external stimulus due to efficient tissue penetration and non-invasiveness. Apart from established clinical ultrasound imaging, ultrasound induces apoptosis in the tumor through thermal effects like hyperthermia or nonthermal effects like cavitation. ${ }^{22}$ Light has common potentials with a magnetic field and ultrasound (such as safe, temporospatial accuracy and less invasiveness). Furthermore, light is tunable in terms of wavelength as there are many useful modalities such as UV light, visible light and NIR light. In the NIR region ranging from 650 to $900 \mathrm{~nm}$, light displays deeper tissue penetration and less photodamage than visible and UV light in vivo applications. ${ }^{23}$ In this review, we outline inorganic nanomaterials used in NIR light-based stimuli-responsive system for PTT. In order to exert maximal therapeutic effects of PTT, we also focus this review on recent applications of inorganic nanomaterials in combinational cancer therapy of PTT and other treatments such as CHT, RT, IT and PDT, as well their antibacterial properties.

\section{Photothermal Therapy in Cancer Treatment}

Compared to UV and visible light, NIR light has photons of a longer wavelength and less energy. This characteristic helps NIR light penetrate deeper into living tissue but NIR photons cannot carry out many photochemical reactions without enough energy. This problem can be solved by mechanisms such as upconversion and two-photon absorption. ${ }^{30}$ Upconversion nanoparticles (UCNP) can transform NIR to visible light and UV light by absorbing two or more photons then converting it into a high energy photon. ${ }^{31}$ Apart from these two mechanisms, the NIR lightresponsive system also follows the photothermal effect mechanism which is at the core for PTT. In this case, lightsensitive nanomaterials can convert NIR light into heat which can be applied to a specific site for hyperthermia. When nanomaterials are applied as nanocarriers for drug delivery systems, heat-activated nanostructures change to release drugs. When nanomaterials are utilized as photothermal agents, heat can damage or ablate tumor cells directly. In order to design a qualified system for PTT, photothermal agents need to satisfy conditions like absorbing NIR light, efficiently converting light to heat and be biocompatible and biodegradable. ${ }^{2}$ Examples of inorganic nanomaterials include AuNPs, ${ }^{27}$ black phosphorus, ${ }^{25}$ carbon nanomaterials and UCNPs. ${ }^{29}$ Various organic nanomaterials also have excellent properties for PTT (such as polymeric materials including polypyrrole (PPy) ${ }^{32}$ and photothermal dyes including perylenediimide, ${ }^{33}$ indocyanine green (ICG) ${ }^{34}$ IR780 ${ }^{35}$ and IR825). ${ }^{36}$

For organic nanomaterials, Yang et al fabricated NIRcontrolled drug-loaded nanoparticles for PTT-CHT. The heat generated from light-absorbable PPy made the nanoparticle size switchable. Upon continuous laser illumination, the nanoparticle swelled to release loaded camptothecin (CPT) and doxorubicin (DOX) and it shrank back to stop releasing CPT and DOX after switching the laser off. ${ }^{32}$ For inorganic nanomaterials, Betzer et al developed immunoconjugated gold nanorods (AuNRs) for selectively targeting and attaching to head and neck cancer (HNC) cells. AuNRs can absorb NIR light and induce hyperthermia to the cancer cells, and can do so in a greater manner than nano spherical particles or larger particles due to their greater surface areas. The results exhibited that PTT with AuNRs effectively decreased tumor size with minimal side effects. ${ }^{37}$ Kang et al fabricated innovative gold nanoparticles/graphene oxide 
(AuNP/GO) hybrid sheets that can attach to the surface of tumor-tropic mesenchymal stem cells (MSC) without being internalized so that cytotoxicity and exocytosis issues can be avoided. Compared to AuNP-internalized MSCs, AuNP/GO sheet-attached MSCs can convert more NIR light to heat in vivo in order to achieve therapeutic efficacy. Moreover, a strong plasmon coupling between AuNPs resulted from the tight packing of AuNPs on the GO sheets which also enhanced the photothermal effect. ${ }^{6}$ Multiple studies also demonstrated the antibacterial effects of inorganic nanomaterials such as AuNPs. ${ }^{13}$ Therefore, as an excellent photothermal agent, AuNPs also possess antibacterial properties. ${ }^{38}$ As stated, due to their weakened immune system, cancer patients are strongly susceptible to infections. The combination of antibacterial and photothermal effects provides a promising method for applying inorganic nanomaterials for cancer treatment.

After converting NIR light to heat, the tumor microenvironment temperature increases. When the temperature is above $45{ }^{\circ} \mathrm{C}$, cancer cells can be killed immediately. However, it inhibits other treatments because it often results in hemorrhage and stasis in tumor vessels. On the contrary, mild hyperthermia around $42-43{ }^{\circ} \mathrm{C}$ can induce cellular damage and increase tumor vessel permeability so that cancer cells are more vulnerable to allied treatments such as CHT or RT. ${ }^{39}$ Because avoiding vascular occlusion can result from higher temperatures, mild hyperthermia can improve nanoparticle uptake by tumors. ${ }^{40}$ An elevated microenvironment temperature improves tumor oxygenation, perfusion and the tendency of cancer cells towards apoptosis. ${ }^{39}$ The above characteristics of mild hyperthermia via nanoparticles support the combination of PTT with other cancer treatments such as PDT, CHT, RT and IT. Furthermore, combination therapies enhance the therapeutic efficacy of individual treatments under synergistic effects which will be discussed in the following paragraphs.

\section{Combination of PTT with CHT, RT, IT and PDT}

PTT has so many advantages, such as high temporospatial control, low cost and minimal invasiveness, and it can also be used alone to eradicate the primary tumor or lymph metastasis in superficial tissues. However, light has a limitation on penetration depth. Even though NIR light can penetrate deeper than UV and visible light, it can only reach around a $3 \mathrm{~cm}$ deep in tissue. ${ }^{41}$ Due to the finite tissue penetration of NIR light in deep tissues, it is a great challenge for PTT alone to eradicate metastatic cancer cells or metastatic nodules in distant organs. ${ }^{42}$ Accordingly, it is necessary to combine PTT with current available therapies for a desired efficacy on cancer metastasis (Figure 1).

\section{Combination Therapy with PTT and CHT}

Multiple studies demonstrated that CHT has several side effects such as drug resistance, intrinsic toxicity to normal cells, and individual behavioral differences in patients. ${ }^{48}$ The problems can be alleviated or solved under the synergistic effect of a combination therapy with PTT. Photothermal agents combined with nanocarriers can deliver CHT drugs to tumors without exposure to normal cells and, thus, reduce the amount of drug needed potentially reducing toxic side effects and the development of chemotherapeutic resistant cancer cells. The release of drugs is limited without NIR irradiation and the drugs are rapidly released when photothermal agents are triggered by NIR irradiation so a lower dosage of drugs is required to achieve the ideal result. ${ }^{49}$ Furthermore, if the release system is enough, intelligent drug-releasing nanoparticles can be immediately ceased when the NIR laser is switched off. ${ }^{50}$ For example, Zhao et al developed an inorganic materialhollow structured polymer-silica nanohybrid (HPSN) as a nanocarrier to deliver the photothermal agent palladium phthalocyanine and chemotherapeutic drug paclitaxel. Due to a surface polyethylene glycol (PEG) ligand coating, most of the off-targeted HPSN could be excreted in 10 days through the hepatobiliary pathway. Fast clearance of nanocarriers efficiently reduced off-target toxicity of the anticancer drugs. The study also showed that the combination of PTT and CHT could inhibit tumor recurrence which could not be achieved by solely using PTT. $^{26}$

Meng et al designed an intelligent $\mathrm{MEO}_{2} \mathrm{MA} @ \mathrm{MEO}_{2}$ MA-co-OEGMA-CuS-DOX composite (G-CuS-DOX). It was composed of $\mathrm{CuS}$ nanoparticles as photothermal agents, DOX as the CHT drug and $\mathrm{MEO}_{2} \mathrm{MA} @ \mathrm{MEO}_{2} \mathrm{MA}-\mathrm{co}-$ OEGMA (G) as a thermosensitive nanogel. Under NIR irradiation, the nanocapsules induced hyperthermia which resulted in shrinkage of the nanogels to release DOX. When NIR irradiation was shut down, both the photothermal and chemotherapeutic effects were interrupted. It was demonstrated that synergistic PTT and CHT exhibited controllable and efficient advantages in in vivo experiments. ${ }^{50}$ Zhang et al fabricated polymer-coated hollow mesoporous silica nanoparticles (HMSNs) encapsulating a photothermal 


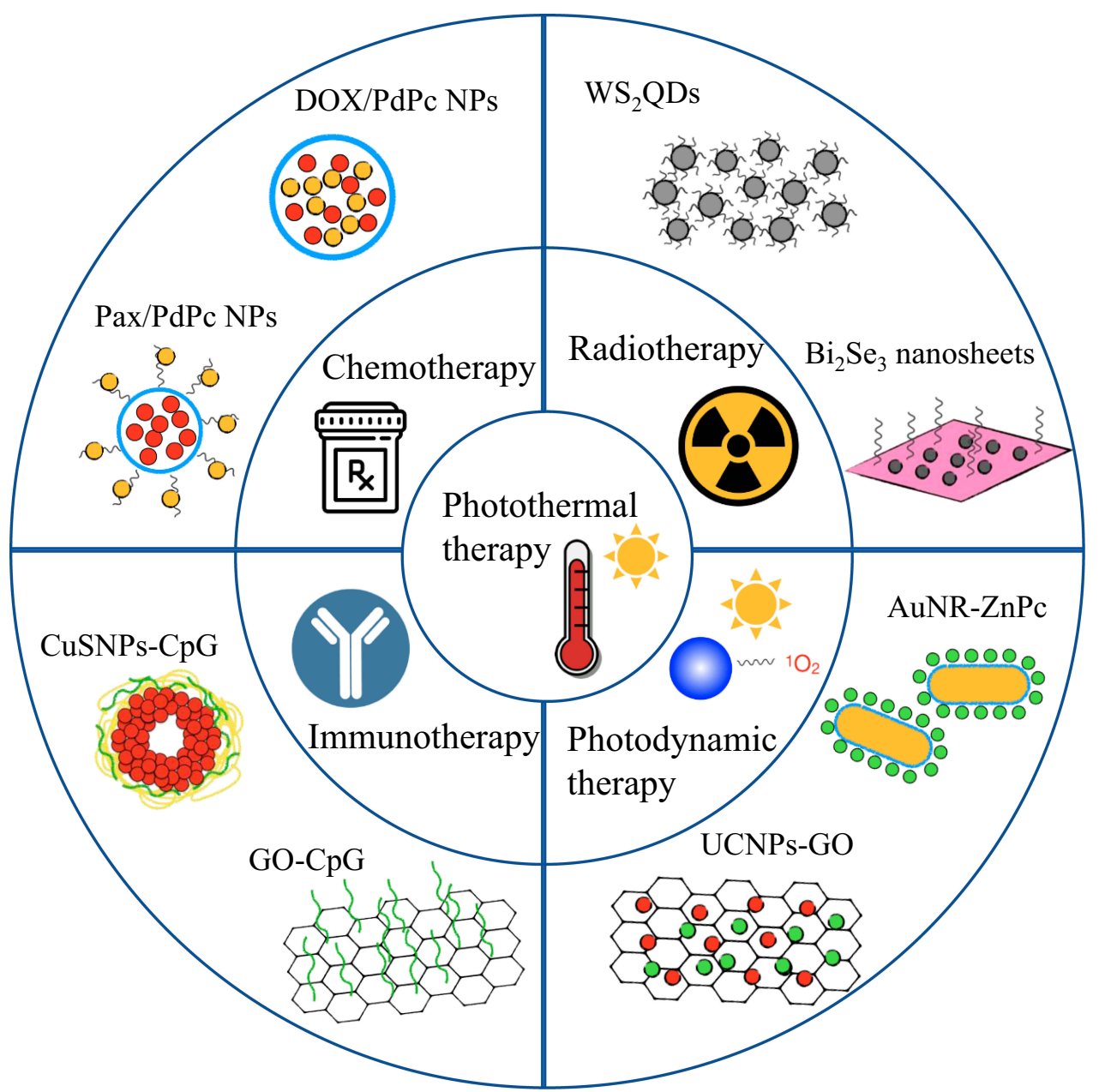

Figure I Schematic illustration for the combinational cancer therapy of photothermal therapy with chemotherapy, radiotherapy, immunotherapy and photodynamic therapy. The outside inorganic nanomaterials are examples of each combinational therapy.

Abbreviations: NPs, nanoparticles; Pax, paclitaxel; DOX, doxorubicin; WS 2 QDs, tungsten sulfide quantum dots; CuSNPs, CuS nanoparticles; CpG, cytosine-guanine; GO, graphene oxide; AuNR, gold nanorod; ZnPc, zinc phthalocyanine; UCNPs, upconversion nanoparticles.

dye IR825 and covalently binding the anticancer drug DOX to the polymers on the outside. DOX was conjugated with a polymer via an acid-labile hydrazine bond which was cleaved in the low $\mathrm{pH}$ tumor microenvironment to release CHT drugs. HMSNs not only reduced the side effects of DOX but it also improved the stability of the organic dye IR825. Furthermore, the combination of DOX and IR825 exhibited therapeutic effects on DOX-resistant A2780/ DOX $^{\mathrm{R}}$ cells. ${ }^{36}$ This represents one of the few studies to combat chemotherapeutic resistant cancer cells. Further, Fang et al developed a multifunctional nanoplatform based on hollow mesoporous carbon nanoparticles (HMCN). HMCN were utilized not only as a nanocarrier to deliver DOX for CHT, but also as a photothermal agent to perform PTT. In addition, the pores from HMCN were capped by graphene quantum dots (GQDs) to prevent the early release of DOX and the surface of HMCN was modified by hyaluronic acid (HA) to increase cancer cell uptake. The morphological changes caused by GQDs can be directly observed in the TEM images (Figure 2A and B). The nanocarrier HAHMCN(DOX)@GQDs under NIR light exhibits excellent in vivo antitumor efficacy - showing an obvious inhibition of tumor growth in mice (Figure 2C and D). ${ }^{51}$ Both Liu et al and Wang et al fabricated nanoplatforms based on hydrogels for the synergistic tumor therapy of PTT and CHT. The solution was injected into the tumor at room temperature and the hydrogel instantaneously formed as the temperature increased to $37^{\circ} \mathrm{C}$. The hydrogel matrix concurrently controlled the release of chemotherapeutic drugs and exhibited a photothermal effect. Their work proved that the designed composite hydrogel was a highly efficient platform for the treatment of tumors. ${ }^{52,53}$ 

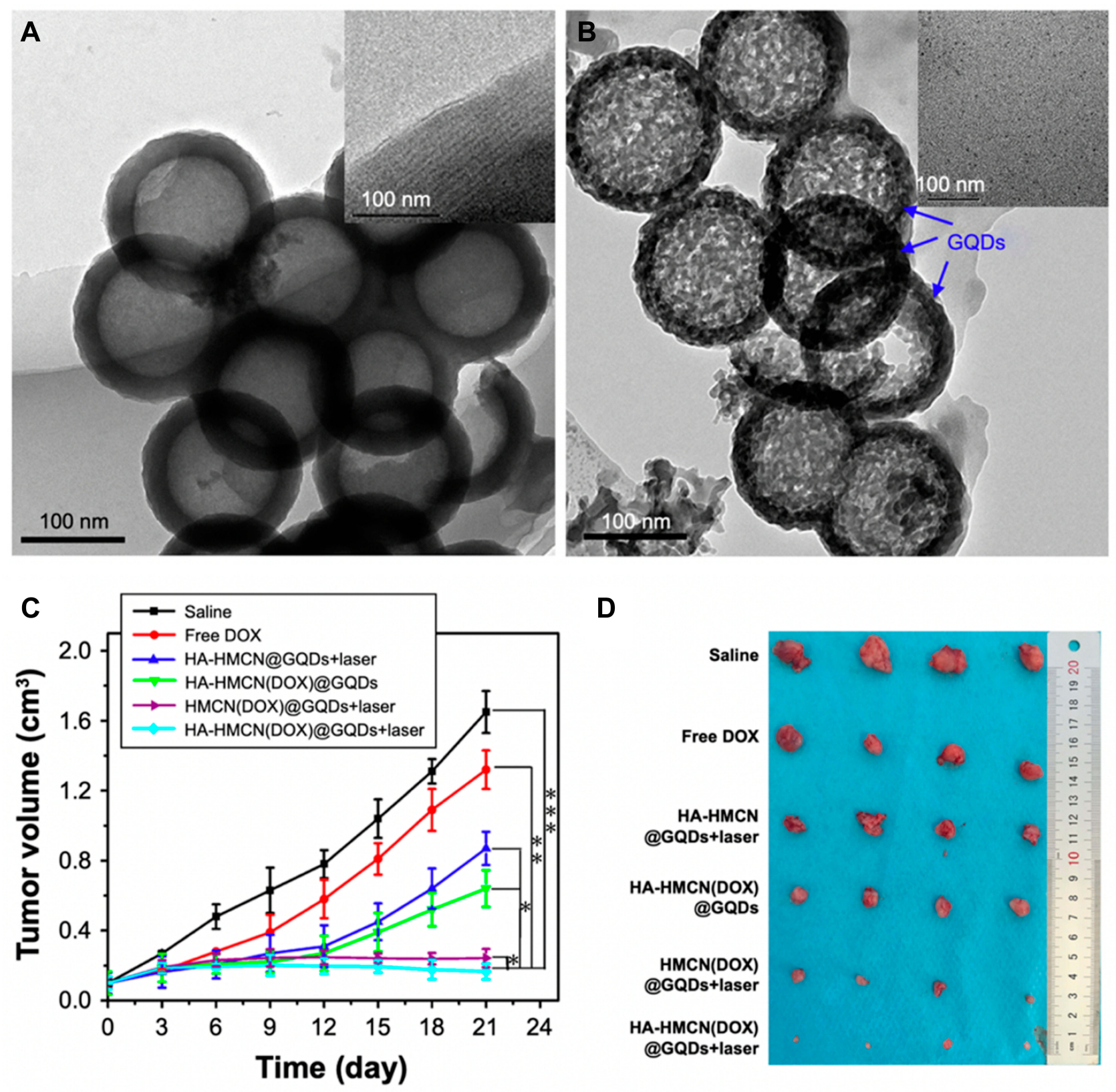

D

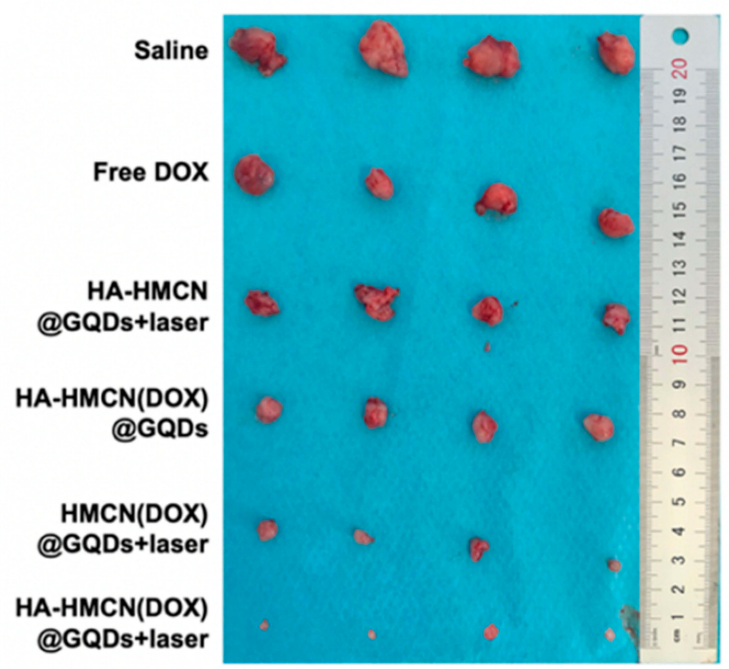

Figure 2 The morphology and in vivo antitumor activity of HA-HMCN(DOX)@GQDs.

Notes: (A) TEM images of HMCN and the mesoporous shell of HMCN (inset). (B) TEM images of HA-HMCN(DOX)@GQDs and GQDs (inset). (C) Relative tumor volume after various treatments $(n=4)$. (D) The photographs of the tumors from each group of mice at the end of therapy on day $2 \mathrm{I}$. ${ }^{\mathrm{p}} \mathrm{p}<0.0 \mathrm{I}$, $* * \mathrm{p}<0.05$, and $* * * \mathrm{p}<0.0 \mathrm{I}$. Adapted from Fang J, Liu Y, Chen Y, Ouyang D, Yang G, Yu T. Graphene quantum dots-gated hollow mesoporous carbon nanoplatform for targeting drug delivery and synergistic chemo-photothermal therapy. Int J Nanomedicine. 2018;13:5991-6007. (c) 2018 Fang et al. ${ }^{51}$

Abbreviations: TEM, transmission electron microscopy; HA, hyaluronic acid; HMCN, hollow mesoporous carbon nanoparticle; DOX, doxorubicin; GQDs, graphene quantum dots.

\section{Combination Therapy of PTT and RT}

The abnormal microenvironment of tumors forms transport barriers limiting the distribution of antitumor agents, from small-molecule anticancer drugs to therapeutic nanomaterials. In order to be delivered into the tumor site, nanoparticles have to overcome transport barriers such as growth-induced solid stress, complex tumor vascular networks, increased interstitial fluid pressure and tortuous interstitial structures. ${ }^{54}$ It has been demonstrated that radiation-treated tumors have increased vessel perfusion, elevated vascular permeability and alleviated interstitial fluid pressure. ${ }^{55}$ Mild hyperthermia induced by PTT also has similar effects including increasing tumor perfusion, vessel permeability and nanoparticle uptake. ${ }^{39}$ 
Furthermore, low-energy long-wavelength NIR light and high-energy short-wavelength radiation compete with each other for further advantages. NIR light has a limited penetration depth while X-ray and $\gamma$-ray radiation do not have depth restrictions. RT displays low therapeutic efficacy for hypoxic cancer cells while PTT efficiently kills this type of cancer cell because hyperthermia can enhance oxygenation status in the tumor. ${ }^{56}$ Thus, the combination treatment of PTT and RT can strengthen the therapeutic efficacy of each alone.

Yong et al designed a multifunctional nanomedicine based on tungsten sulfide quantum dots $\left(\mathrm{WS}_{2} \mathrm{QDs}\right)$ as both radiosensitizers and photothermal agents. Furthermore, $\mathrm{WS}_{2}$ QDs can also be used for dual-modal imaging including X-ray computed tomography (CT) and photoacoustic (PA) imaging because of its high X-ray attenuation ability and strong NIR optical absorbance. The tumor could be precisely located via simultaneous CT/PA imaging and be eradicated by PTT/RT therapy. Besides excellent CT/PA imaging capacity and a PTT/RT synergistic effect, $\mathrm{WS}_{2}$ QDs exhibited remarkable biocompatibility. ${ }^{43}$ Cheng et al fabricated PEG-modified $\mathrm{FeSe}_{2} / \mathrm{Bi}_{2} \mathrm{Se}_{3}$ nanosheets for combined PTT and RT treatment (Figure $3 \mathrm{~A}$ and B). $\mathrm{FeSe}_{2} / \mathrm{Bi}_{2}$ $\mathrm{Se}_{3}-\mathrm{PEG}$ possessed intrinsic properties (such as strong
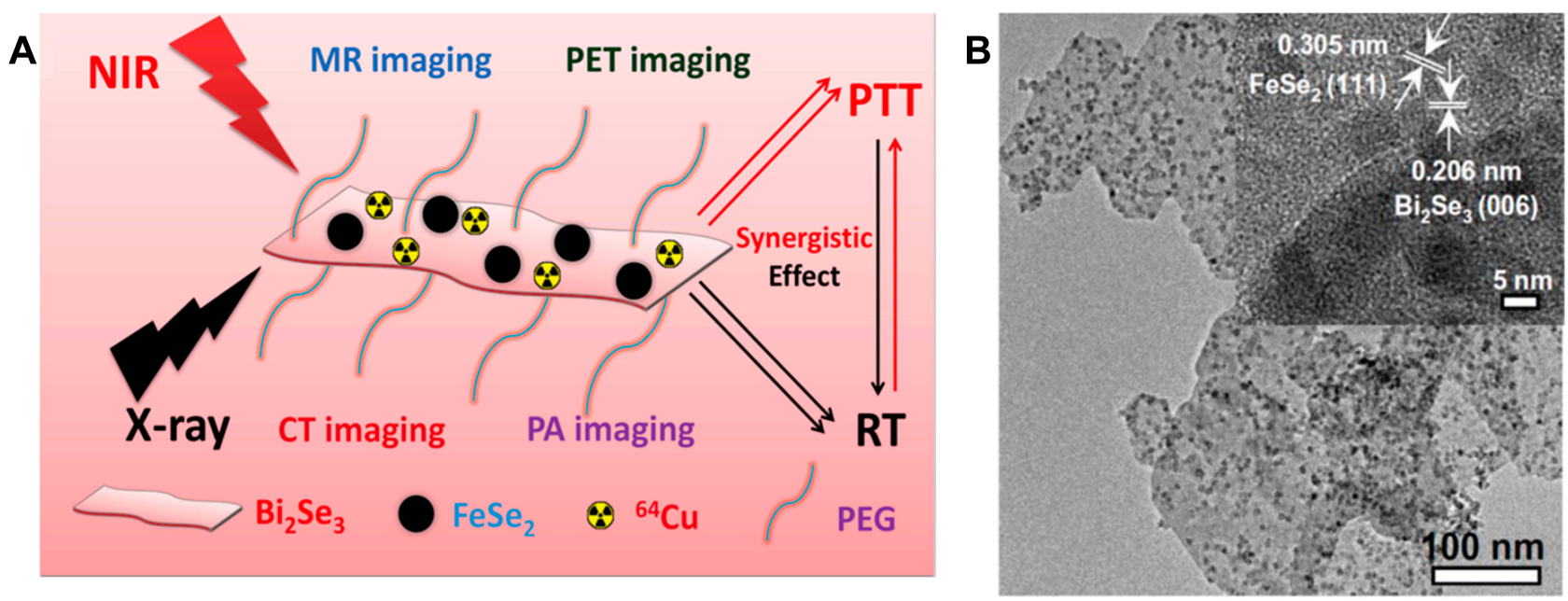

C
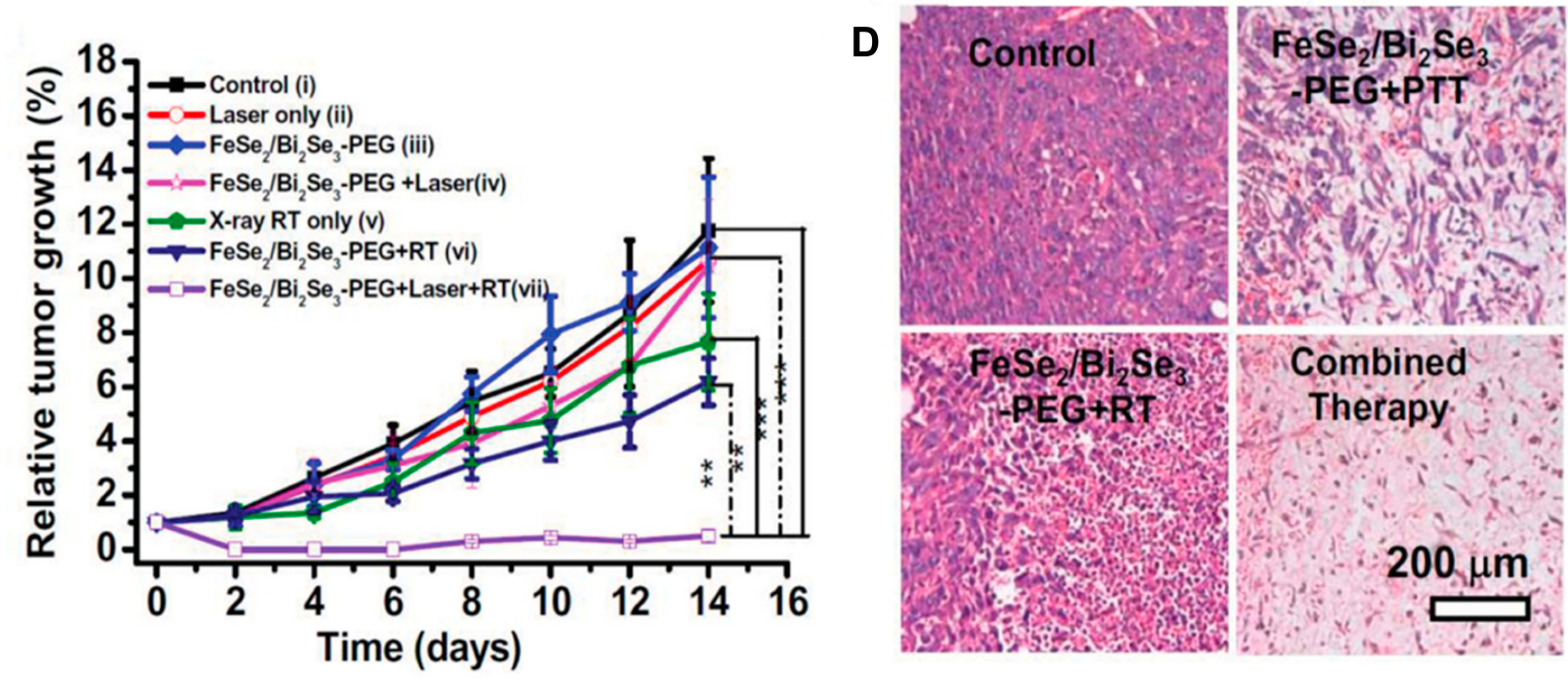

Figure 3 The morphology and in vivo antitumor activity of $\mathrm{FeSe}_{2} / \mathrm{Bi}_{2} \mathrm{Se}_{3}-\mathrm{PEG}$.

Notes: (A) Schematic illustration of the $\mathrm{FeSe}_{2} / \mathrm{Bi}_{2} \mathrm{Se}_{3}$ composite-nanostructure for multimodal imaging and photothermal-radiation combined tumor therapy. (B) The TEM images of $\mathrm{FeSe}_{2} / \mathrm{Bi}_{2} \mathrm{Se}_{3}$ nanostructures and the respective high-resolution TEM images (inset). (C) Relative tumor growth after various treatments ( $\mathrm{n}=5$ ). (D) The images of $\mathrm{H} \& E$ stained tumor slices from different groups of mice treated with PBS, $\mathrm{FeSe}_{2} / \mathrm{Bi}_{2} \mathrm{Se}_{3}-\mathrm{PEG}+\mathrm{NIR}, \mathrm{FeSe}_{2} / \mathrm{Bi}_{2} \mathrm{Se}_{3}-\mathrm{PEG}+\mathrm{RT}$, and FeSe $2 / \mathrm{Bi}_{2} \mathrm{Se}_{3}-\mathrm{PEG}+\mathrm{RT}+\mathrm{NIR}$. The tumors were harvested $2 \mathrm{~d}$ after the treatments were conducted. ${ }^{* *} \mathrm{p}<0.05$ and ${ }^{* * *} \mathrm{p}<0.01$. Reproduced with permission from Cheng L, Shen $\mathrm{S}$, Shi S et al. FeSe2-decorated Bi2Se3 nanosheets fabricated via cation exchange for chelator-free $64 \mathrm{Cu}$-labeling and multimodal image-guided photothermal-radiation therapy. Advanced functional materials. 2016;26(I3):2185-97. (c) 2016 WILEY-VCH Verlag GmbH \& Co. KGaA, Weinheim. ${ }^{44}$

Abbreviations: TEM, transmission electron microscopy; PEG, polyethylene glycol; H\&E, hematoxylin and eosin; PBS, phosphate-buffered saline; NIR, near infrared; RT, radiation therapy. 
X-ray attenuation and high NIR absorption) which are fundamental for RT and PTT, respectively. In addition to being labeled with a radioisotope ${ }^{64} \mathrm{Cu}$, the whole composite nanostructure could be used as a contrast agent for in vivo computer tomography, magnetic resonance (MR), photoacoustic (PA), and positron emission tomography (PET) tetra-modal imaging. $\mathrm{FeSe}_{2} / \mathrm{Bi}_{2} \mathrm{Se}_{3}-\mathrm{PEG}$ displayed remarkable therapeutic effects on tumor destruction and excellent inhibition on tumor growth (Figure 3C and D). ${ }^{44}$ Yi et al developed PEG-modified iodine-131-doped copper sulfide (CuS/[ $\left.\left.{ }^{131} \mathrm{I}\right] \mathrm{I}-\mathrm{PEG}\right)$ nanoparticles for PTT and RT. The nanomaterial took advantage of high NIR optical absorbance for PTT, but it utilized the doped ${ }^{131}$ I-radioactivity for internal CT which is different from external CT used by the above two nanomaterials. Yi's studies demonstrated that $\mathrm{CuS} /\left[{ }^{131} \mathrm{I}\right] \mathrm{I}-\mathrm{PEG}$ offered great synergistic therapeutic efficacy in both in vitro and in vivo experiments. Importantly, the combined PTT and RT could inhibit cancer metastasis and prolong animal survival when applied to lymph nodes for assisting in the surgical removal of primary tumors. ${ }^{57}$

\section{Combination Therapy of PTT and IT}

Compared to gold-standard cancer treatments such as surgery, CHT and RT, IT is an emerging treatment through training or stimulating the host immunological systems to kill tumor cells. ${ }^{58}$ IT has shown remarkable promises, but it still has many limitations like high cost, immunotoxicity and large individual variations. ${ }^{59}$ Some nanomaterialbased therapies (such as PTT) could strengthen the immune response in IT. Hyperthermia triggered by PTT can not only kill cancer cells directly but can also induce the release of tumor-associated antigens which are essential for antigen processing and presentation. If IT and PTT are combined into one treatment, IT could perform a more therapeutic efficiency against tumors with the help of the released antigens and immunoadjuvants. ${ }^{60}$

Guo et al designed chitosan-coated hollow $\mathrm{CuS}$ nanoparticles (HCuSNPs) with oligodeoxynucleotides (ODNs) containing cytosine-guanine $(\mathrm{CpG})$ motifs as the immunoadjuvants. $\mathrm{CpG}$ ODNs are efficient immunological modulators. Nanoparticles can enhance their in vivo stability, pharmacokinetic and biodistribution properties and intracellular uptake for being recognized by TLR9. ${ }^{28}$ NIR irradiation triggers the disintegration and reassembly of nanoparticles to form chitosan- $\mathrm{CpG}$ nanocomplexes with high tumor retention and intracellular uptake. It was demonstrated that HCuSNPs-CpG exhibited more potent immune responses and remarkable synergistic anticancer effects than IT or PTT alone. After NIR irradiation, nanoparticles can be quickly eliminated from the body because of their biodegradability. ${ }^{45}$ Tao et al utilized PEG and polyethyleneimine (PEI) dual-polymer-functionalized graphene oxide (GO) as the carrier for delivering CpG. GOPEG-PEI-CpG nanocomplexes can remarkably improve the production of proinflammatory cytokines and strengthen the immunostimulatory activity under NIR irradiation. Moreover, immune responses are efficiently improved because the photothermal effect of GO accelerates intracellular transportation of $\mathrm{CpG}$ ODNs. ${ }^{46}$

\section{Combination Therapy of PTT and PDT}

Both PTT and PDT are treatments as a result of lightresponsive nanomaterials, but PTT depends on hyperthermia to kill cancer cells while PDT depends on reactive oxygen species (ROS) to kill tumor cells. Even though their therapeutic mechanisms are different, PTT and PDT have similar light triggering conditions. ${ }^{61}$ Thus, PTT and PDT can work independently and complementarily under the same NIR laser irradiation. ${ }^{62}$ For example, Wang et al fabricated an ICG-based cisplatin polyprodrug nanoplatform in which the concurrent photodynamic and photothermal therapy exhibited excellent performance. Upon cellular uptake in lysosomes, ICG could induce local hyperthermia and ROS under NIR light irradiation and it could also assist the escape of cisplatin prodrugs into the reductive cytosol by damaging lysosomes. ${ }^{34}$ In addition, the combination treatment of PDT can benefit from pathogen inhibition. Liu et al developed azonia-containing polyelectrolytes with extraordinary performance for ROS generation for antibacterial applications. ${ }^{63}$

But back to inorganic nanomaterials, Tham et al reported novel nanomaterial-zinc phthalocyanine $(\mathrm{ZnPc})$ anchored silica-coated gold nanorods (AuNRs) for combinational PDT and PTT. Anchored ZnPc is a PDT precursor used to produce singlet oxygen. AuNR is a photothermal agent exhibiting localized surface plasmon resonance (LSPR), which then, hyperthermia induced by AuNRs can improve blood flow and oxygenation to and in the tumor, further improving PDT. ${ }^{64}$ In Tham's study, both $\mathrm{ZnPc}$ and AuNR were excited by NIR light leading to hyperthermia and ROS at the tumor site for synergistic PTT and PDT. ${ }^{47}$ Kalluru et al fabricated a GO-PEG-folate nanocomposite to exert photothermal and photodynamic therapeutic effects on tumors. GO (as the core of the nanomaterial) possesses intrinsic photothermal properties and can generate 
a singlet oxygen for PDT. Kalluru's team showed that cellular death was caused by PDT and PTT in vitro experiments and they confirmed that the combination treatment was more effective than PDD or PTT alone using in vivo experiments. ${ }^{65}$ Wang et al developed a multifunctional nanoplatform through covalently grafting upconversion nanoparticles (UCNPs) with nanographene oxide (NGO) and loading $\mathrm{ZnPc}$ on the surface of NGO. In UCNPs$\mathrm{NGO} / \mathrm{ZnPc}$, ZnPc was used as a photosensitizer for PDT and UCNPs were utilized as photothermal agents for PTT. Then, NGO worked as a carrier for $\mathrm{ZnPc}$ and UCNPs because of its high NIR absorption and large surface area. According to this study, the combined treatment showed significantly greater performance on cancer therapy. ${ }^{29}$ In addition, both Wang et al and Zhang et al also utilized UCNPs for enhancing the efficacy of PDT. Under NIR light excitation, UCNPs can emit UV-vis light then further excite photosensitizers to generate ROS. ${ }^{31,66}$

\section{Conclusion}

In summary, several inorganic nanomaterial-based combinational cancer therapies are discussed in this review combining PTT/CHT, PTT/RT, PTT/IT and PTT/PDT. The most important advantages that such inorganic nanoparticle provide are improved therapeutic efficacy and safety. In each combinational therapy, two cancer treatments work independently and complementarily and the synergistic effects due to each is unique. For combined PTT/CHT, nanomaterials lower the dosage of anticancer drugs and protect normal cells from the intrinsic toxicity of the drugs. It also makes the drug release system more controllable and efficient. For the combined PTT/RT, both hyperthermia and radiation can change the tumor microenvironment to correspond to each other's treatment. PTT exerts better performance on radiation-treated tumors and RT works better on hypoxic cancer cells with hyperthermia-induced oxygenation. For combined PTT and IT, tumor-associated antigens induced by PTT and immunoadjuvants coupled on nanomaterials result in strengthening of the immune system. For combined PTT and PDT, the same NIR light irradiation can simultaneously activate PTT and PDT to produce heat and ROS. Besides the above combinational therapies, the combination with chemodynamic therapy is also a promising approach. Due to the high level of intracellular $\mathrm{H}_{2} \mathrm{O}_{2}$ in tumor cells, it is possible to produce cytotoxic hydroxyl radicals $(\cdot \mathrm{OH})$ by the catalysis of metal ions provided by nanoplatforms. Because the nanoplatform is specific to the tumor microenvironment, the efficiency of the combination therapy can be enhanced. ${ }^{67}$

Despite the fact that inorganic nanoparticle combinational therapies have favorable properties, they still need further investigation. Recent studies in this review demonstrated the application of nanomaterials for such purposes using in vitro and in vivo experiments. However, it is a long way for them to be used in clinical trials and preclinical data should be further optimized. Furthermore, combinational therapies exhibit enhanced therapeutic effects, but the underlying mechanisms of synergistic effects (and how they can be further optimized) require further investigation, such as combined PDT and IT properties. The therapeutic efficacy of inorganic nanomaterials can be further enhanced by utilizing their antibacterial effects. It is a promising way to protect cancer patients from infections during cancer treatments. The development of nanomaterials makes the multifunctional platform possible. In the future, antibacterial effects could be set further as an intrinsic property requirement for developing nanomaterials for cancer treatment.

Although this review presents some promise aspects for nanoparticles to simultaneously treat cancer cells and bacteria, much more work is needed especially on the antibacterial side. Furthermore, it is also possible to combine PTT with other cancer treatments (such as chemodynamic therapy ${ }^{67}$ and nanoCRISPR ${ }^{68}$ ) together to promote the synergistic effects necessary for treating cancer. Inorganic nanomaterials have demonstrated exceptional accomplishments in biomedical research. The application of such materials in biomedicine requires more actuation and stability due to the complex environment of living organisms. Tumor targeting and biodegradability of the inorganic nanomaterial cannot be ignored in the case of toxicity caused by the material. Clearly, although showing much promise, more efforts are required for achieving additional advances in the development of inorganic nanomaterials for anti-cancer biomedicine.

\section{Acknowledgments}

This project was supported by a seed grant from the Wenzhou Institute, University of Chinese Academy of Sciences (WIUCASQD2019004), Key Laboratory of Orthopaedics of Zhejiang Province (Grant No: ZJGK1804Y), Wenzhou Scientific Research Project (Y20190136), Natural Science Foundation of Zhejiang Province (LY19C100002), National Natural Science Foundation of China (81972892, 31971260) and Zhejiang Province Natural Science Fund for Distinguished Young Scholars (LR20C100001). 


\section{Disclosure}

The authors report no conflicts of interest in this work.

\section{References}

1. Siegel RL, Miller KD, Jemal A. Cancer statistics, 2019. CA Cancer J Clin. 2019;69(1):7-34. doi:10.3322/caac.v69.1

2. Chen H, Zhao Y. Applications of light-responsive systems for cancer theranostics. ACS Appl Mater Interfaces. 2018;10(25):21021-21034. doi:10.1021/acsami.8b01114

3. Deng J, Xun X, Zheng W, et al. Sequential delivery of bismuth nanoparticles and doxorubicin by injectable macroporous hydrogels for combined anticancer kilovoltage X-ray radio-and chemo-therapy. J Mater Chem B. 2018;6(47):7966-7973. doi:10.1039/C8TB02284D

4. Muntimadugu E, Kommineni N, Khan W. Exploring the potential of nanotherapeutics in targeting tumor microenvironment for cancer therapy. Pharmacol Res. 2017;126:109-122. doi:10.1016/j.phrs.20 17.05.010

5. Iyer AK, Khaled G, Fang J, Maeda H. Exploiting the enhanced permeability and retention effect for tumor targeting. Drug Discov Today. 2006;11(17-18):812-818. doi:10.1016/j.drudis.2006.07.005

6. Kang S, Lee J, Ryu S, et al. Gold nanoparticle/graphene oxide hybrid sheets attached on mesenchymal stem cells for effective photothermal cancer therapy. Chem Mater. 2017;29(8):3461-3476. doi:10.1021/ acs.chemmater.6b05164

7. Liu J, Wang C, Wang X, et al. Mesoporous silica coated single-walled carbon nanotubes as a multifunctional light-responsive platform for cancer combination therapy. Adv Funct Mater. 2015;25(3):384-392. doi:10.1002/adfm.201403079

8. Raju GSR, Dariya B, Mungamuri SK, et al. Nanomaterials multifunctional behavior for enlightened cancer therapeutics. Semin Cancer Biol. 2019. doi:10.1016/j.semcancer.2019.08.013

9. Overchuk M, Zheng G. Overcoming obstacles in the tumor microenvironment: recent advancements in nanoparticle delivery for cancer theranostics. Biomaterials. 2018;156:217-237. doi:10.1016/j. biomaterials.2017.10.024

10. Deng J, Xu S, Hu W, Xun X, Zheng L, Su M. Tumor targeted, stealthy and degradable bismuth nanoparticles for enhanced X-ray radiation therapy of breast cancer. Biomaterials. 2017;154:24-33. doi:10.1016/j.biomaterials.2017.10.048

11. Albanese A, Tang PS, Chan WC. The effect of nanoparticle size, shape, and surface chemistry on biological systems. Annu Rev Biomed Eng. 2012;14:1-16. doi:10.1146/annurev-bioeng-071811150124

12. Jokerst JV, Lobovkina T, Zare RN, Gambhir SS. Nanoparticle PEGylation for imaging and therapy. Nanomedicine. 2011;6 (4):715-728. doi:10.2217/nnm.11.19

13. Hajipour MJ, Fromm KM, Ashkarran AA, et al. Antibacterial properties of nanoparticles. Trends Biotechnol. 2012;30(10):499-511. doi:10.1016/j.tibtech.2012.06.004

14. Huh AJ, Kwon YJ. "Nanoantibiotics": a new paradigm for treating infectious diseases using nanomaterials in the antibiotics resistant era. JControl Release. 2011;156(2):128-145. doi:10.1016/j.jconrel.2011.07.002

15. Dai Y, Xu C, Sun X, Chen X. Nanoparticle design strategies for enhanced anticancer therapy by exploiting the tumour microenvironment. Chem Soc Rev. 2017;46(12):3830-3852. doi:10. 1039/C6CS00592F

16. Li HJ, Du JZ, Liu J, et al. Smart superstructures with ultrahigh $\mathrm{pH}$-sensitivity for targeting acidic tumor microenvironment: instantaneous size switching and improved tumor penetration. ACS Nano. 2016;10(7):6753-6761. doi:10.1021/acsnano.6b02326

17. Qian C, Yu J, Chen Y, et al. Light-activated hypoxia-responsive nanocarriers for enhanced anticancer therapy. Adv Mater. 2016;28 (17):3313-3320. doi:10.1002/adma.201505869
18. Li L, Nurunnabi N, Lee YK, Huh KM. GSH-mediated photoactivity of pheophorbide a-conjugated heparin/gold nanoparticle for photodynamic therapy. $J$ Control Release. 2013;171(2):241-250. doi:10. 1016/j.jconrel.2013.07.002

19. Horsman MR, Vaupel P. Pathophysiological basis for the formation of the tumor microenvironment. Front Oncol. 2016;6:66. doi:10.33 89/fonc. 2016.00066

20. Mura S, Nicolas J, Couvreur P. Stimuli-responsive nanocarriers for drug delivery. Nat Mater. 2013;12(11):991-1003. doi:10.1038/nmat3776

21. Sun XY, Liu MC, Chen XL, et al. A dual-targeted nucleic acid moiety decorated SPION nanoparticles for chemo-photodynamic synergistic therapy. J Lumin. 2019;209:387-397. doi:10.1016/j. jlumin.2019.02.019

22. Zhao YZ, Du LN, Lu CT, Jin YG, Ge SP. Potential and problems in ultrasound-responsive drug delivery systems. Int J Nanomedicine. 2013;8:1621-1633. doi:10.2147/IJN.S43589

23. Karimi M, Sahandi Zangabad P, Baghaee-ravari S, Ghazadeh M, Mirshekari H, Hamblin MR. Smart nanostructures for cargo delivery: uncaging and activating by light. $J$ Am Chem Soc. 2017;139 (13):4584-4610. doi:10.1021/jacs.6b08313

24. Fan $\mathrm{CH}$, Cheng $\mathrm{YH}$, Ting $\mathrm{CY}$, et al. Ultrasound/magnetic targeting with SPIO-DOX-microbubble complex for image-guided drug delivery in brain tumors. Theranostics. 2016;6(10):1542-1556. doi:10.71 $50 /$ thno. 15297

25. Shao J, Xie H, Huang $H$, et al. Biodegradable black phosphorus-based nanospheres for in vivo photothermal cancer therapy. Nat Commun. 2016;7:12967. doi:10.1038/ncomms12967

26. Zhao L, Yuan W, Tham HP, et al. Fast-clearable nanocarriers conducting chemo/photothermal combination therapy to inhibit recurrence of malignant tumors. Small. 2017;13(29):1700963. doi:10.10 02/smll.v13.29

27. Wang R, Deng J, He D, et al. PEGylated hollow gold nanoparticles for combined X-ray radiation and photothermal therapy in vitro and enhanced CT imaging in vivo. Nanomedicine. 2019;16:195-205. doi:10.1016/j.nano.2018.12.005

28. Wilson KD, de Jong SD, Tam YK. Lipid-based delivery of CpG oligonucleotides enhances immunotherapeutic efficacy. Adv Drug Deliv Rev. 2009;61(3):233-242. doi:10.1016/j.addr.2008.12.014

29. Wang Y, Wang H, Liu D, Song S, Wang X, Zhang H. Graphene oxide covalently grafted upconversion nanoparticles for combined NIR mediated imaging and photothermal/photodynamic cancer therapy. Biomaterials. 2013;34(31):7715-7724. doi:10.1016/j.biomaterials.20 13.06.045

30. Zhang F, editor. Upconversion nanoparticles for light-activated therapy. In: Photon Upconversion Nanomaterials. Heidelberg: Springer; 2015:285-341.

31. Zhao N, Wu B, Hu X, Xing D. NIR-triggered high-efficient photodynamic and chemo-cascade therapy using caspase-3 responsive functionalized upconversion nanoparticles. Biomaterials. 2017; 141:40-49. doi:10.1016/j.biomaterials.2017.06.031

32. Yang J, Zhai S, Qin $\mathrm{H}$, Yan $\mathrm{H}$, Xing $\mathrm{D}, \mathrm{Hu}$ X. NIR-controlled morphology transformation and pulsatile drug delivery based on multifunctional phototheranostic nanoparticles for photoacoustic imaging-guided photothermal-chemotherapy. Biomaterials. 2018; 176:1-12. doi:10.1016/j.biomaterials.2018.05.033

33. Jiao Y, Liu K, Wang G, Wang Y, Zhang X. Supramolecular free radicals: near-infrared organic materials with enhanced photothermal conversion. Chem Sci. 2015;6(7):3975-3980. doi:10.1039/C5SC01167A

34. Wang W, Liang G, Zhang W, Xing D, Hu X. Cascade-promoted photo-chemotherapy against resistant cancers by enzyme-responsive polyprodrug nanoplatforms. Chem Mater. 2018;30(10):3486-3498. doi:10.1021/acs.chemmater.8b01149

35. Chen Y, Li Z, Wang H, et al. IR-780 loaded phospholipid mimicking homopolymeric micelles for near-ir imaging and photothermal therapy of pancreatic cancer. ACS Appl Mater Interfaces. 2016;8 (11):6852-6858. doi:10.1021/acsami.6b00251 
36. Zhang Y, Ang CY, Li M, Tan SY, Qu Q, Zhao Y. Polymeric prodrug grafted hollow mesoporous silica nanoparticles encapsulating near-infrared absorbing dye for potent combined photothermal-chemotherapy. ACS Appl Mater Interfaces. 2016;8 (11):6869-6879. doi:10.1021/acsami.6b00376

37. Betzer O, Ankri R, Motiei M, Popovtzer R. Theranostic approach for cancer treatment: multifunctional gold nanorods for optical imaging and photothermal therapy. J Nanomater. 2015;2015:1-7. doi:10.1155/ 2015/646713

38. Penders J, Stolzoff M, Hickey DJ, Andersson M, Webster TJ. Shapedependent antibacterial effects of non-cytotoxic gold nanoparticles. Int J Nanomedicine. 2017;12:2457-2468. doi:10.2147/IJN

39. Fukumura D, Jain RK. Tumor microenvironment abnormalities: causes, consequences, and strategies to normalize. J Cell Biochem. 2007;101(4):937-949. doi:10.1002/(ISSN)1097-4644

40. Kong G, Braun RD, Dewhirst MW. Characterization of the effect of hyperthermia on nanoparticle extravasation from tumor vasculature. Cancer Res. 2001;61(7):3027-3032.

41. Wu S, Butt H-J. Near-infrared photochemistry at interfaces based on upconverting nanoparticles. Phys Chem Chem Phys. 2017;19 (35):23585-23596. doi:10.1039/C7CP01838J

42. Zou L, Wang H, He B, et al. Current approaches of photothermal therapy in treating cancer metastasis with nanotherapeutics. Theranostics. 2016;6(6):762-772. doi:10.7150/thno.14988

43. Yong Y, Cheng X, Bao T, et al. Tungsten sulfide quantum dots as multifunctional nanotheranostics for in vivo dual-modal image-guided photothermal/radiotherapy synergistic therapy. ACS Nano. 2015;9(12):12451-12463. doi:10.1021/acsnano.5b05825

44. Cheng L, Shen S, Shi S, et al. FeSe2-decorated Bi2Se3 nanosheets fabricated via cation exchange for chelator-free $64 \mathrm{Cu}$-labeling and multimodal image-guided photothermal-radiation therapy. Adv Funct Mater. 2016;26(13):2185-2197. doi:10.1002/adfm.201504810

45. Guo L, Yan DD, Yang D, et al. Combinatorial photothermal and immuno cancer therapy using chitosan-coated hollow copper sulfide nanoparticles. ACS Nano. 2014;8(6):5670-5681. doi:10.1021/ nn5002112

46. Tao Y, Ju E, Ren J, Qu X. Immunostimulatory oligonucleotides-loaded cationic graphene oxide with photothermally enhanced immunogenicity for photothermal/immune cancer therapy. Biomaterials. 2014;35 (37):9963-9971. doi:10.1016/j.biomaterials.2014.08.036

47. Tham HP, Chen H, Tan YH, et al. Photosensitizer anchored gold nanorods for targeted combinational photothermal and photodynamic therapy. Chem Commun. 2016;52(57):8854-8857. doi:10.1039/ C6CC03076A

48. Peer D, Karp JM, Hong S, Farokhzad OC, Margalit R, Langer R. Nanocarriers as an emerging platform for cancer therapy. Nat Nanotechnol. 2007;2(12):751. doi:10.1038/nnano.2007.387

49. Li Y, He D, Tu J, et al. The comparative effect of wrapping solid gold nanoparticles and hollow gold nanoparticles with doxorubicin-loaded thermosensitive liposomes for cancer thermo-chemotherapy. Nanoscale. 2018;10(18):8628-8641. doi:10.1039/C7NR09083H

50. Meng Z, Wei F, Wang R, et al. NIR-laser-switched in vivo smart nanocapsules for synergic photothermal and chemotherapy of tumors. Adv Mater. 2016;28(2):245-253. doi:10.1002/ adma.201502669

51. Fang J, Liu Y, Chen Y, Ouyang D, Yang G, Yu T. Graphene quantum dots-gated hollow mesoporous carbon nanoplatform for targeting drug delivery and synergistic chemo-photothermal therapy. Int J Nanomedicine. 2018;13:5991-6007. doi:10.2147/IJN

52. Liu Y, Xi Y, Zhao J, et al. Preparation of therapeutic-laden konjac hydrogel for tumor combination therapy. Chem Eng J. 2019; 375:122048.
53. Zheng Y, Wang W, Zhao J, et al. Preparation of injectable temperature-sensitive chitosan-based hydrogel for combined hyperthermia and chemotherapy of colon cancer. Carbohydr Polym. 2019;222:115039. doi:10.1016/j.carbpol.2019.115039

54. Chauhan VP, Stylianopoulos T, Boucher Y, Jain RK. Delivery of molecular and nanoscale medicine to tumors: transport barriers and strategies. Annu Rev Chem Biomol Eng. 2011;2:281-298. doi:10.1146/annurev-chembioeng-061010-114300

55. Jain RK, Martin JD, Stylianopoulos T. The role of mechanical forces in tumor growth and therapy. Annu Rev Biomed Eng. 2014;16:321-346. doi:10.1146/annurev-bioeng-071813-105259

56. Liu Y, Liu Y, Bu W, et al. Radiation-/hypoxia-induced solid tumor metastasis and regrowth inhibited by hypoxia-specific upconversion nanoradiosensitizer. Biomaterials. 2015;49:1-8. doi:10.1016/j. biomaterials.2015.01.028

57. Yi X, Yang K, Liang C, et al. Imaging-guided combined photothermal and radiotherapy to treat subcutaneous and metastatic tumors using iodine-131-doped copper sulfide nanoparticles. Adv Funct Mater. 2015;25(29):4689-4699. doi:10.1002/adfm.201502003

58. Sheng WY, Huang L. Cancer immunotherapy and nanomedicine. Pharm Res. 2011;28(2):200-214. doi:10.1007/s11095-010-0258-8

59. Chen Q, Xu L, Liang C, Wang C, Peng R, Liu Z. Photothermal therapy with immune-adjuvant nanoparticles together with checkpoint blockade for effective cancer immunotherapy. Nat Commun. 2016;7:13193. doi:10.1038/ncomms13193

60. Dong X, Liang J, Yang A, Qian Z, Kong D, Lv F. Fluorescence imaging guided CpG nanoparticles-loaded IR820-hydrogel for synergistic photothermal immunotherapy. Biomaterials. 2019;209: 111-125. doi:10.1016/j.biomaterials.2019.04.024

61. Cai Y, Liang P, Tang Q, et al. Diketopyrrolopyrrole-triphenylamine organic nanoparticles as multifunctional reagents for photoacoustic imaging-guided photodynamic/photothermal synergistic tumor therapy. ACS Nano. 2017;11(1):1054-1063. doi:10.1021/acsnano. $6 \mathrm{~b} 07927$

62. Wang S, Huang P, Nie L, et al. Single continuous wave laser induced photodynamic/plasmonic photothermal therapy using photosensitizer-functionalized gold nanostars. Adv Mater. 2013;25 (22):3055-3061. doi:10.1002/adma.v25.22

63. Liu X, Li M, Han T, et al. In situ generation of azonia-containing polyelectrolytes for luminescent photopatterning and superbug killing. J Am Chem Soc. 2019;141(28):11259-11268. doi:10.1021/ jacs.9b04757

64. Liu Y, Liu Y, Bu W, et al. Hypoxia induced by upconversion-based photodynamic therapy: towards highly effective synergistic bioreductive therapy in tumors. Angew Chem Int Ed. 2015;54(28):8105-8109. doi:10.1002/anie.201500478

65. Kalluru P, Vankayala R, Chiang CS, Hwang KC. Nano-graphene oxide-mediated In vivo fluorescence imaging and bimodal photodynamic and photothermal destruction of tumors. Biomaterials. 2016;95:1-10. doi:10.1016/j.biomaterials.2016.04.006

66. Zhang D, Wen L, Huang R, Wang H, Hu X, Xing D. Mitochondrial specific photodynamic therapy by rare-earth nanoparticles mediated near-infrared graphene quantum dots. Biomaterials. 2018;153:14-26. doi:10.1016/j.biomaterials.2017.10.034

67. Wu C, Wang S, Zhao J, et al. Biodegradable Fe(III)@WS $\mathrm{W}_{2}-\mathrm{PVP}$ nanocapsules for redox reaction and TME-enhanced nanocatalytic, photothermal, and chemotherapy. Adv Funct Mater. 2019;29(26). doi:10.1002/adfm.201901722

68. Chen X, Chen Y, Xin H, Wan T, Ping Y. Near-infrared optogenetic engineering of photothermal nanoCRISPR for programmable genome editing. Proc Natl Acad Sci. 2020;117:2395-2405. doi:10.1073/ pnas. 1912220117 


\section{Publish your work in this journal}

The International Journal of Nanomedicine is an international, peerreviewed journal focusing on the application of nanotechnology in diagnostics, therapeutics, and drug delivery systems throughout the biomedical field. This journal is indexed on PubMed Central, MedLine, CAS, SciSearch ${ }^{\circledR}$, Current Contents ${ }^{\circledR} /$ Clinical Medicine,
Journal Citation Reports/Science Edition, EMBase, Scopus and the Elsevier Bibliographic databases. The manuscript management system is completely online and includes a very quick and fair peer-review system, which is all easy to use. Visit http://www.dovepress.com/ testimonials.php to read real quotes from published authors.

Submit your manuscript here: https://www.dovepress.com/international-journal-of-nanomedicine-journal 